\title{
Engineering analysis of ITER In-Vessel Viewing System guide tube
}

\author{
Natalia Casal ${ }^{a, *}$, Philip Bates ${ }^{b}$, Ottó Bede $^{c}$, Carlo Damiani $^{\mathrm{b}}$, Gregory Dubus ${ }^{\mathrm{b}}$, \\ Hassan Omran ${ }^{c}$, Jim Palmer ${ }^{a}$, Adrian Puiu ${ }^{b}$, Roger Reichle ${ }^{\mathrm{a}}$, Alejandro Suárez ${ }^{\mathrm{a}}$, \\ Christopher Walker ${ }^{\mathrm{a}}$, Michael Walsh ${ }^{\mathrm{a}}$ \\ ${ }^{a}$ ITER Organization, Route de Vinon sur Verdon, St Paul-lez-Durance, France \\ ${ }^{\mathrm{b}}$ Fusion for Energy, Barcelona, Spain \\ c Oxford Technologies Ltd., Abingdon, UK
}

\section{H I G H L I G H T S}

- Conceptual design of IVVS Loads action on IVVS Dominant loads.

- Seismic and baking conditions.

- No active cooling needed for IVVS.

- IVVS requires additional support points to avoid excessive deformation.

\section{Keywords:}

ITER

IVVS

\begin{abstract}
A B S T R A C T
The In Vessel Viewing System (IVVS) will be one of the essential machine diagnostic systems at ITER to provide information about the status of in-vessel and plasma facing components and to evaluate the dust inside the Vacuum Vessel. The current design consists of six scanning probes and their deployment systems, which are placed in dedicated ports at the divertor level. These units are located in resident guiding tubes $10 \mathrm{~m}$ long, which allow the IVVS probes to go from their storage location to the scanning position by means of a simple straight translation. Moreover, each resident tube is supported inside the corresponding Vacuum Vessel and Cryostat port extensions, which are part of the primary confinement barrier. As the Vacuum Vessel and the Cryostat will move with respect to each other during operation (especially during baking) and during incidents and accidents (disruptions, vertical displacement events, seismic events), the structural integrity of the resident tube and the surrounding vacuum boundaries would be compromised if the required flexibility and supports are not appropriately assured. This paper focuses on the integration of the present design of the IVVS into the Vacuum Vessel and Cryostat environment. It presents the adopted strategy to withstand all the main interfacing loads without damaging the confinement barriers and the corresponding analysis supporting it.
\end{abstract}

\section{Introduction}

The in vessel viewing system (IVVS) will be one of the essential diagnostic systems at ITER to provide information about the status of in-vessel and plasma facing components and to participate in the strategy of dust evaluation inside the Vacuum Vessel [1]. To do that, six units will be placed in dedicated ports at the divertor level, Fig. 1. Each unit comprises a viewing/metrology probe mounted on a deployment arm that can be inserted into the ITER Vacuum Vessel

\footnotetext{
* Corresponding author.

E-mail address: natalia,casal@iter.org (N. Casal).
}

(VV) at positions between the divertor outer target baffle and the lower outer blanket modules. This operation can be done between discharges or during maintenance periods with or without magnetic field at operational temperature $\left(120^{\circ} \mathrm{C}\right)$ and under vacuum or atmospheric pressure conditions.

These units are located in resident guiding tubes $10 \mathrm{~m}$ long, which allow the IVVS probes to go from their storage location to the scanning position by means of a simple straight translation. Moreover, each resident tube is supported inside the correspondent Vacuum Vessel and Cryostat port extensions, which are part of the primary confinement barrier. This paper is specifically focused on the analysis of the engineering of the guide tube system of each unit. 


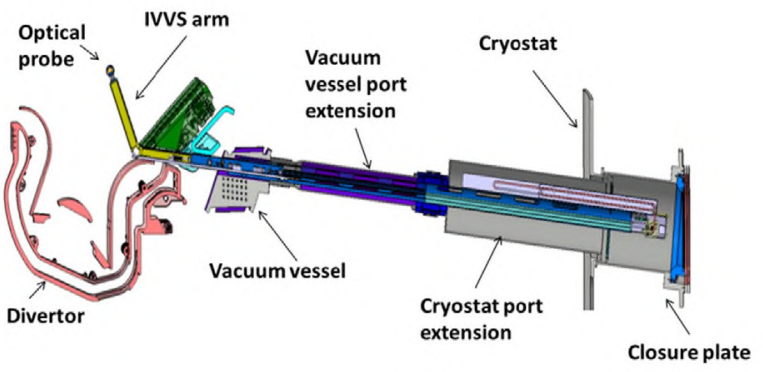

Fig. 1. Overview of IVVS in its dedicated port.

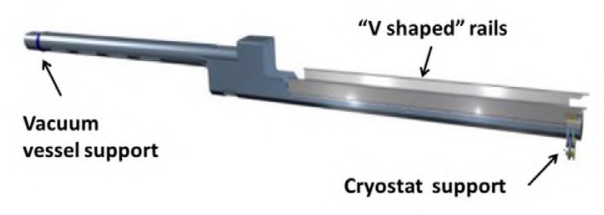

Fig. 2. Guide tube system.

\section{Description of the system}

The IVVS active components are installed in a guide resident tube which is a passive system supported at the rear part of the dedicated cryostat port extension by a mechanism allowing two rotational degrees of freedom in order to avoid experiencing any bending loads due to the relative motion between the vacuum vessel and the cryostat. The front of the guide tube is supported in the VV penetration by a barreled ring which allows it to swivel and slide within the VV penetration hole.

The guide tube, Fig. 2, will be equipped with "V" shaped rails where the so called IVVS cartridge will dock. The cartridge assembly is the removable part of the system which can be transported through a cask to the ITER hot cell. It is approximately $4.2 \mathrm{~m}$ long and weighs $1080 \mathrm{~kg}$. It comprises a mobile assembly that carries the optical probe which is translated by a push chain system. The guide tube will also carry a moveable shield system that protects the probe closing the line of sight with the vacuum vessel during operation, when the probe is retracted [2].

\section{Load evaluation}

\subsection{Load evaluation}

In this chapter, the main loads considered for the IVVS structural integrity evaluation are summarized.

\subsubsection{Dead weigh}

The total weight of the different IVVS components reaches $2615 \mathrm{~kg}$. The resident tube weights $884 \mathrm{~kg}$, the cartridge is $1082 \mathrm{~kg}$, and mobile assembly is $213 \mathrm{~kg}$. Finally, the static shield assembly weighs $436 \mathrm{~kg}$.

\subsubsection{Electromagnetic loads}

The IVVS may be used in fields of up to 8 Tesla to allow for invessel inspection after plasma pulses. While the Magnetic fields are static in normal operation conditions, movement of the IVVS components in the field may cause eddy currents within those components. However, no design driving loads are expected as it is assumed that the slow speed of movement of IVVS components and the materials chosen will generate negligible EM forces.

Nevertheless, Tokamak structures are occasionally exposed to rapidly changing magnetic fields caused by abrupt termination of the plasma current. These are known as "plasma disruptions" and

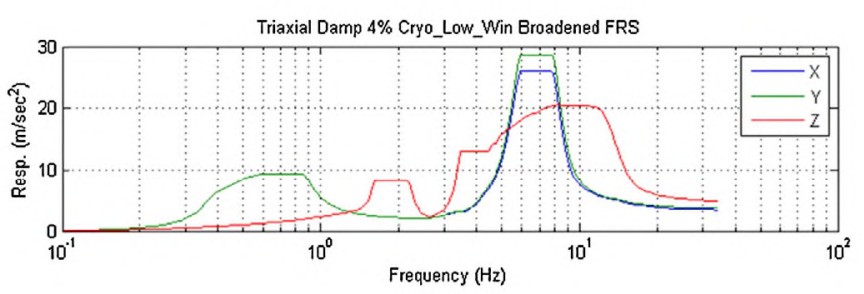

Fig. 3. SL-2 Floor Response Spectra, $\mathrm{m} / \mathrm{s}^{2}$.

"vertical displacement events". They occur on the time scale of a few ms up to seconds. The changing magnetic fields induce eddy currents in metallic structures which then interact with the remaining magnetic fields to usually generate very large forces and torques in passive structures. For the IVVS, preliminary calculations indicate that the total forces acting on each component due to these events are quite small. Forces above $1 \mathrm{kN}$ are present only on the ports (vessel side and cryostat side) and on the stub. Forces on the IVVS components reach a maximum of $364 \mathrm{~N}$ on the resident guide tube, $109 \mathrm{~N}$ the guide tube joint. However, during the vertical displacement events, the vacuum vessel will be accelerated in such a way that, during a short time, strong inertial loads may be developed. How these loads will affect the IVVS guiding tube will be estimated in detail at further stages of the design. As the center of gravity of the IVVS is fully at the cryostat side, the effect of these inertial loads is not be a design driver.

\subsubsection{Seismic loads}

The strongest seismic event foreseen in ITER is called SL-2, and is also known as the Safe Shutdown Earthquake (SSE). This event will be an extremely unlikely event but nevertheless, it shall be demonstrated that all safety functions are maintained. Other seismic event that needs consideration is the so called SMHV (Séismes Maximaus Historiquement Vraisemblables) that will be the most penalizing earthquake liable to occur over a period of about 1000 years, assumed to be equal to SL- 2 multiplied by 0.73 . Finally, also a likely seismic event, called SL- 1 with a probability in the order of $10^{-2}$ per year needs to be taken into account. It is assumed to be equal to $1 / 3$ of SL-2. In the following Fig. 3, the Floor Response Spectra of a representative point at the cryostat support of the IVVS (where the IVVS center of gravity is positioned) is shown for the SL2 scenario. $Z$ represents the vertical direction while $X$ and $Y$ are the radial and toroidal directions respectively.

The worst loading combination cases for the IVVS guiding tube correspond with the seismic events produced during the baking of the vacuum vessel which corresponds with unlikely events but nevertheless, the integrity of the VV and cryostat ports needs to be guaranteed.

\subsubsection{Thermal loads}

During plasma pulses, the thermal loads on the IVVS are dominated by nuclear loads. Electron Cyclotron Resonance Heating (ECRH) can reach the IVVS system during the breakdown of the plasma where no absorption of the microwave power is expected during $5.5 \mathrm{~s}$. During that phase of operation, the IVVS is in the parked position with the moveable shield block in place. $3 \mathrm{KW}$ are expected during $5.5 \mathrm{~s}$ to impinge on the opening of the IVVS penetration to the vacuum vessel as conservative approximation. The temperature increase in the tube is expected to be negligible.

According to neutronics simulations the maximum nuclear heating expected in the front part of the resident tube is approximately $0.04 \mathrm{~W} / \mathrm{cm}^{3}$ for the inductive scenario with $500 \mathrm{MW}$ of fusion power. 


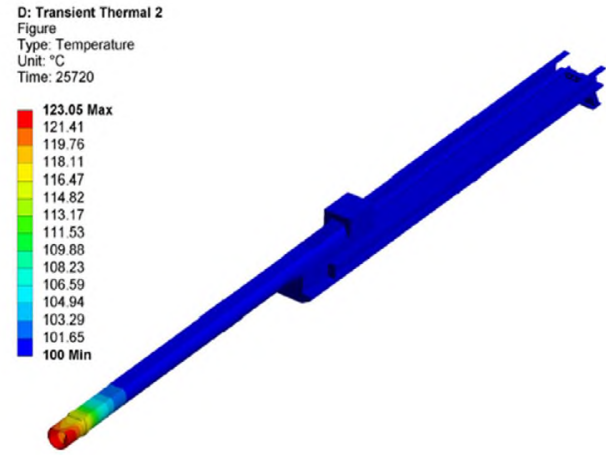

Fig. 4. Map of temperature during nuclear heating.

\subsubsection{Interfacing loads}

The displacements between the vacuum vessel and the cryostat during the different operational states will be one of the worst loads for the IVVS guiding tube. The larger displacement will occur during the VV baking where it will move $40 \mathrm{~mm}$ radially and $20 \mathrm{~mm}$ upwards. Seismic events will produce displacements in the order of 2-10 $\mathrm{mm}$ while the expected displacements during vertical displacement events in the VV would reach about $20 \mathrm{~mm}$.

\section{Engineering analysis}

A number of thermal and structural analyses were carried out by means of ANSYS code [3] to determine the thermal and structural behavior of the IVVS resident tube (made of austenitic stainless steel SS316LN).

\subsection{Thermal analysis}

A thermal transient analysis was performed to determine the response, in terms of maximum temperature and the elapsed time to reach it, under two loading scenarios: a cyclic nuclear heating loading condition during inductive scenario, and baking conditions of VV.

During the inductive scenario, pulses of about $450 \mathrm{~s}$ will take place with a minimum repetition time of $1800 \mathrm{~s}$. The ramp up of the pulse is estimated to be up to $50 \mathrm{~s}$ whereas the ramp down about $100 \mathrm{~s}$.

Under these conditions, and just considering radiation to atmosphere with no conduction to VV (vacuum vessel very close at $100^{\circ} \mathrm{C}$ ) with emissivity 0.3 of external surfaces, a maximum temperature of $123^{\circ} \mathrm{C}$ was obtained in the front part of the tube after 15 cycles where stationary conditions are obtained, Fig. 4 . Then, the IVVS resident tube will be a passively cooled structure.

For the second loading condition, a baking scenario was simulated by considering the surrounding ports at $200^{\circ} \mathrm{C}$ with emissivity of 0.3 . After $14 \mathrm{~h}$, the resident tube surfaces reached $200^{\circ} \mathrm{C}$ from its initial normal operation temperature. This temperature will allow the required outgassing of the surfaces without using active baking water.

\subsection{Structural analysis}

These analyses were carried out to determine the response of the tube assembly under SL-2 event during baking. The structural model considered frictional contacts between the guide tube and the VV.A combination of frictional, frictionless and bonded contacts simulates the hinge fixed to Cryostat port, Fig. 5.

Only considering the self-weight of the resident tube and its internal components simulated as point masses, clashes with the ports have been found at the joint (bellows) of $\mathrm{V}$ with cryostat

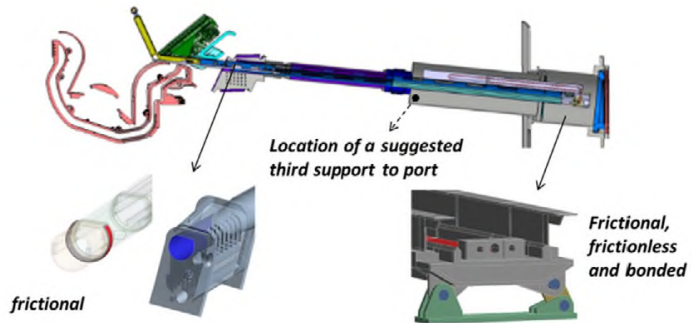

Fig. 5. Supports to the VV and Cryostat.

port where the minimum clearance is about $10 \mathrm{~mm}$. It suggests unacceptable displacements during seismic events. An implementation of a third support seems to become necessary and has been considered as a potential solution, Fig. 5. This support was added in the central part of the Guide Tube modeled as fixed support. In this way, the maximum displacement of the Tube under its self-weight is reduced to $2.1 \mathrm{~mm}$.

A modal analysis has been performed in order to estimate critical natural frequencies of the assembly with the third fixing point. It was found that the frequency of $12.3 \mathrm{~Hz}$ was a critical one. Others are in the range $16-45 \mathrm{~Hz}$. A static equivalent analysis was performed with seismic accelerations corresponding to this critical. A safety margin of 1.5 was considered to take into account that the structure does not behave as a single vibration mode system. Under this loading condition maximum tube displacements are $9.9 \mathrm{~mm}$, Fig. 6. It suggests that the third support should be a rail type one along the cryostat port.

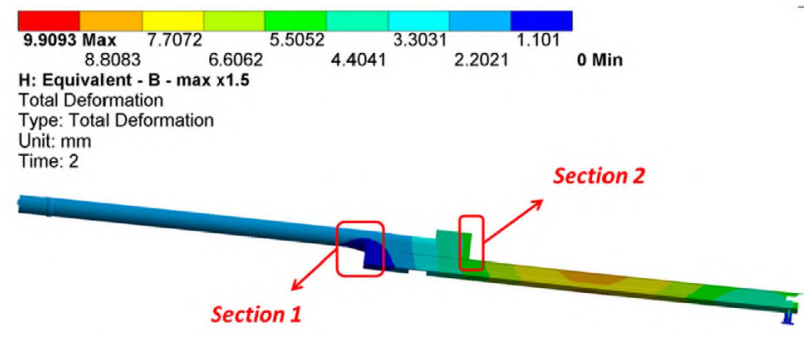

Fig. 6. Displacements in $\mathrm{mm}$ under $\mathrm{SL}-2$. (For interpretation of the references to color in text near the reference citation, the reader is referred to the web version of this article.)

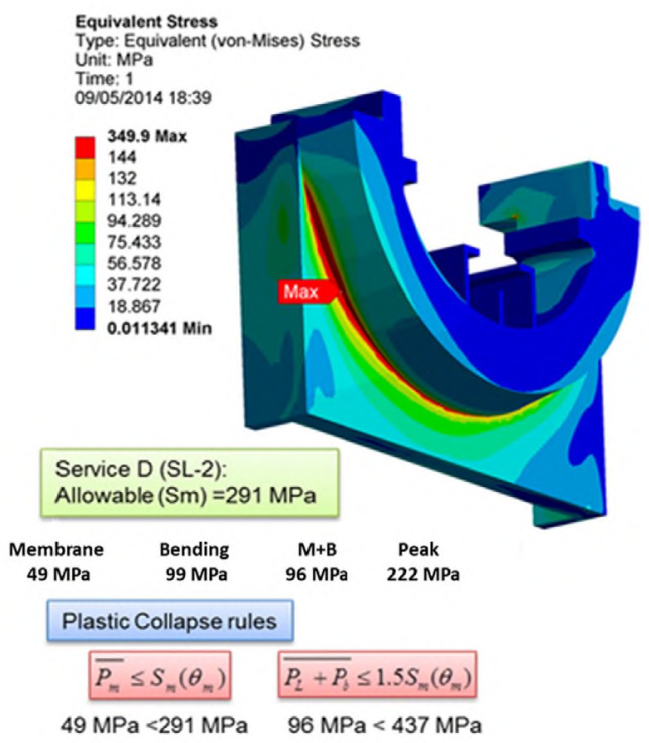

Fig. 7. Stress linearization for section $1, \mathrm{SL}-2$. 


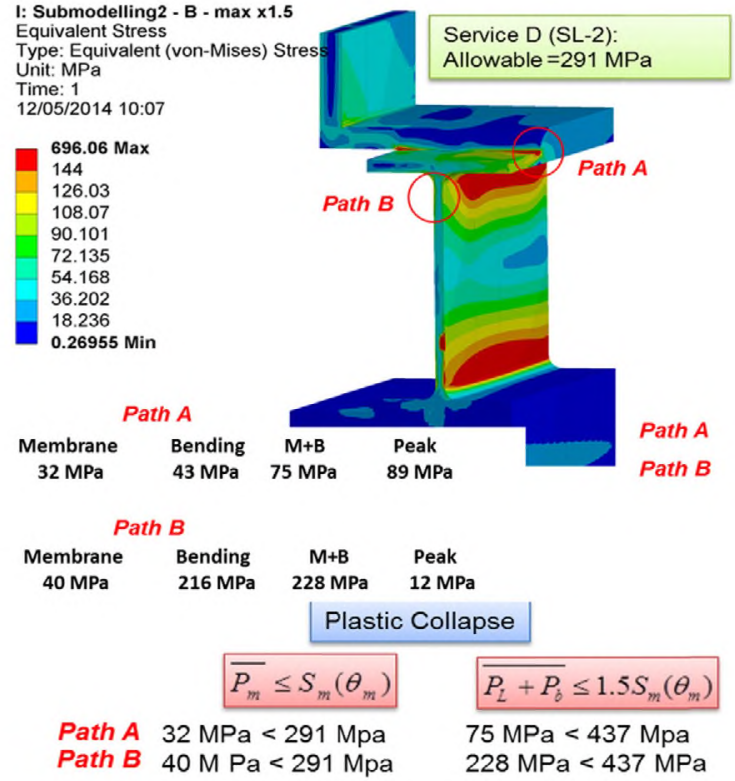

Fig. 8. Stress linearization for section 2, SL-2.

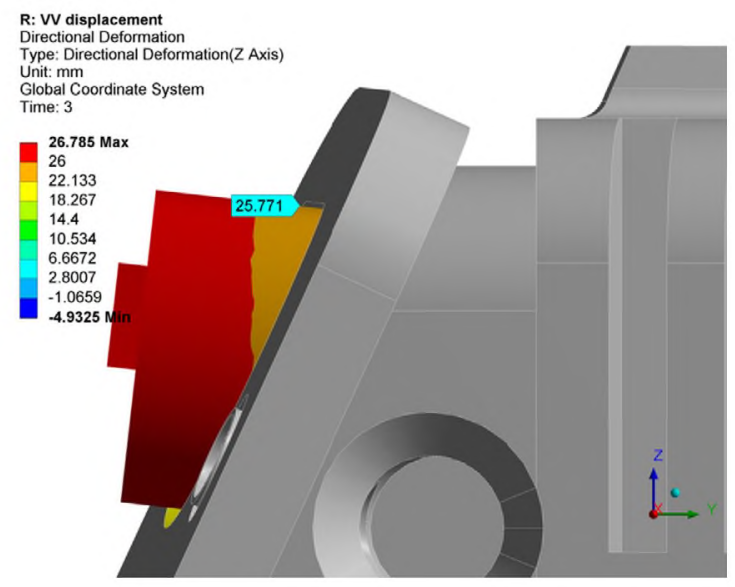

Fig. 9. Displacement of tube tip during VV baking.

Under this situation, 2 sections (red lines in Fig. 6) of the resident tube were found critical ones and a detailed assessment based on sub-modeling was carried out, Figs. 7 and 8 . In section 1, protection against plastic collapse under SL-2 event is guaranteed following the RCC-MR 2007 Service D allowable stress at $240^{\circ} \mathrm{C}$. Even under the consideration of this event as SMHV (corresponding to RCC-MR 2007 Service Level C) no plastic collapse is expected.

In section 2 , the protection against plastic collapse under SL2 event is guaranteed following the RCC-MR 2007 Service Level D. However, stress values do not meet the Service Level C criterion and dedicated analysis will be required in future to analyze the SMHV event (accelerations 0.73 times lower). As a first approximation, considering a factor of 0.73 to SL-2 results, it can be concluded that
Service Level C criterion is also fulfilled although the safety margin would need to be enlarged. For that reason, this section has been identified as a hotspot to be optimized in further design stages.

A third analysis has been performed by considering the interface displacements that the VV (gray structure in Fig. 9) will impose to the tube tip during baking conditions.

In this case, the VV will experience displacements of $20 \mathrm{~mm}$ upwards and $40 \mathrm{~mm}$ radially. As the tube support at the VV allows the radial movement, the tip of the tube will enter into the $\mathrm{VV}$. As shown in Fig. 9, the tube structure will deform during the vertical movement and some points will suffer displacements in the range of $25 \mathrm{~mm}$. At this position, clearance between tube and the $\mathrm{VV}$ is only $5 \mathrm{~mm}$ and the risk of clash is evident. Modifying the geometry toward a tray shape, opening the upper part of the tube, was found the proper solution which is fully compatible with the deployment of the arm.

\section{Conclusions}

The IVVS resident tube and its internal components are in its conceptual design phase. The main loads coming from the ITER environment have been identified and discussed in this paper. Seismic events and baking conditions will dominate all other load conditions. Preliminary analyses have been done to identify the weak points that will need further optimization in the next iteration.

Preliminary thermal transient analysis demonstrates that active cooling is not needed for IVVS. The IVVS guiding tube structure supported only at its ends would deform too much clashing with surrounding ports. An additional support is considered a solution to this problem. This has been preliminary assessed. The behavior of the guide tube under seismic events and the imposed displacements by the VV is promising with the third additional support. Only few sections of the structure would need slight reinforcements during the next design phase. Also the tip of the guiding tube would need certain optimization in order to avoid clashes with the VV port stub during baking conditions. Nevertheless, this can be easily solved with a tray type resident tube.

\section{Disclaimer}

The views and opinions expressed herein do not necessarily reflect those of the ITER Organization.

\section{Acknowledgments}

All ITER Organization teams, domestic agencies and associated companies that did possible the work presented in this paper.

\section{References}

[1] C. Neri, ITER in-vessel viewing system design and assessment activities, Fusion Eng. Des. $86(9-11)(2011)$.

[2] G. Dubus, Conceptual design finalization of the ITER In vessel viewing system and Metrology System (IVVS)", Fusion Eng. Des. 88 (October (9-10)) (2013).

[3] ANSYS $^{\mathfrak{B}}$, Release 14.0, Help System, Structural Analysis Guide, ANSYS, Inc. 